\title{
LIFETIME MEASURMENTS OF EXCITED STATES IN NEUTRON-RICH FISSION FRAGMENTS*
}

\author{
T.W. HaGen ${ }^{\mathrm{a}}$, L. Grente ${ }^{\mathrm{b}}$, A. GörGen ${ }^{\mathrm{a}}$, M.-D. SAlsaC ${ }^{\mathrm{b}}$ \\ W. Korten ${ }^{\mathrm{b}}$, F. FArGet ${ }^{\mathrm{c}}$, T. Braunroth ${ }^{\mathrm{d}}$, B. Bruyneel $^{\mathrm{b}}$

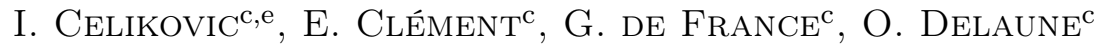

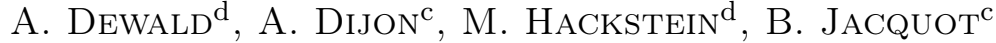

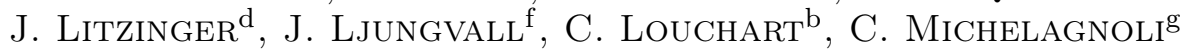

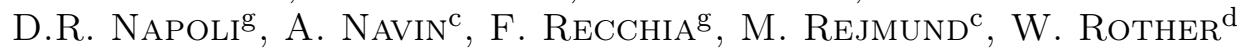

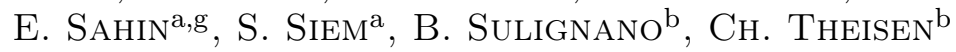 \\ J.J. VALIEnTE-Dobon ${ }^{g}$ \\ ${ }^{a}$ Department of Physics, University of Oslo, Norway \\ ${ }^{b}$ IRFU, Service de Physique Nucléaire, CEA Saclay, France \\ ${ }^{\mathrm{c}}$ Grand Accélérateur National d'Ions Lourds (GANIL), Caen, France \\ ${ }^{\mathrm{d}}$ Institut für Kernphysik, Universität zu Köln, Germany \\ e Institute of Nuclear Sciences 'Vinca', University of Belgrade, Serbia \\ ${ }^{\mathrm{f}} \mathrm{CSNSM}$, IN2P3, Orsay, France \\ ${ }^{g}$ Laboratori Nazionali di Legnaro, INFN, Italy
}

(Received December 18, 2015)

Lifetimes of short-lived excited states in a wide range of neutron-rich fission fragments were measured using the recoil distance Doppler shift (RDDS) technique, which was applied to fusion-fission reactions in inverse kinematics for the first time. The fission fragments were identified eventby-event in mass, charge, and atomic number using the VAMOS magnetic spectrometer at GANIL. Gamma rays originating from the fission fragments were measured with the EXOGAM array of Ge Clover detectors around the target position. Using a degrader, the change in the Doppler shift of the $\gamma$ ray allows the application of the RDDS method. Details of the experimental technique will be discussed and the status for the ongoing analysis for odd-mass yttrium isotopes will be presented.

DOI:10.5506/APhysPolB.47.903

\section{Introduction}

In order to further our understanding of nuclear structure, it is essential to acquire experimental observables that can be compared to theoretical

* Presented at the XXXIV Mazurian Lakes Conference on Physics, Piaski, Poland, September 6-13, 2015. 
predictions. Good testing grounds for this are areas where nuclei undergo sudden, non-linear changes. One area of the nuclear chart where dramatic changes can be observed is the region of $Z \sim 40$ and $N \sim 60$ nuclei. Here, a sudden onset of deformation occurs from neutron number 58 to 60 . This is evidenced by a steep drop of the energy of the first $2^{+}$states and a corresponding increase of the $B(\mathrm{E} 2)$ values in even-even $\mathrm{Sr}$ and $\mathrm{Zr}$ nuclei. Furthermore, a sudden increase of the difference in the mean-square charge radii is observed for the isotopic chains of $\mathrm{Rb}, \mathrm{Sr}, \mathrm{Y}, \mathrm{Zr}$, and $\mathrm{Nb}$ isotopes [1, 2]. Nuclei in this transitional region, where spherical and deformed configurations cross in energy, exhibit shape coexistence. Experimental evidence for shape coexistence comes from the observation of low-lying excited $0^{+}$states with strong E0 transitions [3, 4]. The nuclei with $Z \geq 42$ show a more gradual transition from near spherical to deformed shapes, and it is expected that triaxiality becomes increasingly important.

Electromagnetic transition rates are important observables for a quantitative understanding of nuclear shapes and can be used to benchmark nuclear structure theories. There are two complementary methods to determine electromagnetic matrix elements for short-lived excited states: Coulomb excitation and lifetime measurements. Coulomb excitation of ${ }^{96,98} \mathrm{Sr}$ has yielded spectroscopic quadrupole moments and reduced transition probabilities for various low-lying states. The results have given direct evidence for the crossing of the near-spherical and well-deformed configuration between ${ }^{96} \mathrm{Sr}$ and ${ }^{98} \mathrm{Sr}$ [5]. A similar Coulomb excitation experiment for the ${ }^{97,99} \mathrm{Rb}$ isotopes indicates that the sudden shape transition is also present in the $\mathrm{Rb}$ chain and that the deformation for Rb nuclei above $N=60$ is similar to the other strongly deformed nuclei in the region [6]. The objective of the present experiment was to provide electromagnetic transition rates from lifetime measurements for a wide range of nuclei in this particular region of the nuclear landscape where shape transitions and shape coexistence occur.

The mass region has been extensively studied using, for example, $\beta$-delayed spectroscopy with on-line mass separators. With fast electronic timing techniques, it is possible to measure lifetimes greater than a few tens of picoseconds [7]. Studies using spontaneous fission sources and high-fold $\gamma$-ray coincidences have provided extensive level schemes [8] where, consistent with strongly deformed nuclei, regular rotational bands are seen for the $N=60$ nuclei. In some cases, it was possible to measure lifetimes of excited states using Doppler-shift techniques with fission sources [9]. However, in such experiments, it is not possible to identify the fission fragments directly. Instead, coincidences with known $\gamma$ rays from the isotope of interest are used for identification. Our approach to use fusion-fission reactions in inverse kinematics has the advantage that the fission fragments are produced with high velocity, facilitating an event-by-event identification in a magnetic 
spectrometer and, at the same time, inducing large Doppler shifts that can be utilized to measure short lifetimes down to the picosecond range using the RDDS method.

Here, we discuss experimental details of this new variant of the RDDS technique and, as an example of the results obtained in this measurement, we discuss preliminary results from the ongoing analysis to extract lifetimes

of excited states in the ground-state band of ${ }_{39}^{99} \mathrm{Y}_{60}$. Similar to neighboring Y and $\mathrm{Nb}$ isotopes with $N \geq 60$, the coupling of the odd proton with the deformed even-even core results in a strongly coupled (deformation aligned) rotational band built on the ground state [10]. Combining the measurement of the lifetime of excited states with the M1/E2 branching ratio of their decay, it is possible to extract absolute M1 and E2 reduced transition probabilities. The analysis of the $B(\mathrm{M} 1)$ and $B(\mathrm{E} 2)$ values with the particle-rotor model provides information on the $g$-factor of the odd proton and the quadrupole moment of the deformed ${ }^{98} \mathrm{Sr}$ core.

\section{Experimental details}

The experiment was performed at GANIL using the EXOGAM HPGe detector array coupled to the magnetic spectrometer VAMOS [11, 12]. A ${ }^{238} \mathrm{U}$ beam of $6.2 \mathrm{~A} \mathrm{MeV}$ impinged on a ${ }^{9} \mathrm{Be}$ target of $2.3 \mathrm{mg} / \mathrm{cm}^{2}$ thickness. The reaction leaves the compound nucleus ${ }^{247} \mathrm{Cm}$ at a relatively high excitation energy of approximately $45 \mathrm{MeV}$, causing the system to fission on a time scale that is short compared to the electromagnetic decay process of the fission fragments. Since shell effects are largely washed out at this excitation energy, the fragment distribution is more symmetric compared to neutron-induced or spontaneous fission. Due to the inverse kinematics, the fission fragments are strongly forward focused, which facilitates their identification in mass, charge, and atomic number in the large-acceptance spectrometer VAMOS.

The spectrometer consists of two quadrupole and one dipole magnet and was rotated to an angle of $20^{\circ}$ with respect to the beam axis. Two multiwire PPAC detectors at the entrance of the first quadrupole and in the focal plane provide a time-of-flight measurement, and two drift chambers provide the position and angle of the fragments in the focal plane, allowing the reconstruction of the trajectories. The energy loss and total energy of the fragments are measured with a segmented ionization chamber and a wall of 40 Si detectors, respectively. The VAMOS spectrometer with its focal plane detectors makes it possible to identify the fission fragments on an event-byevent basis. Evaluating the energy loss $\Delta E$ of the fragments in the ionization chamber as a function of their total energy $E$ provides identification of the atomic number $Z$, as shown in Fig. 1. 

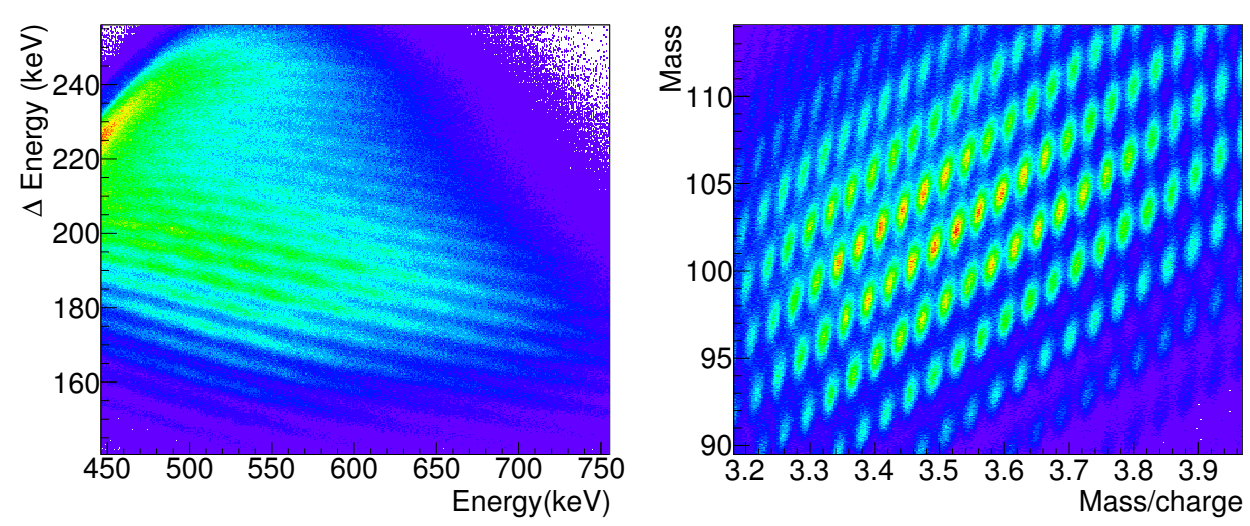

Fig. 1. Left: Plot of the energy loss $\Delta E$ in the ionization chamber versus the total energy $E$ deposited in the ionization chamber and the Si detectors. Each horizontal locus corresponds to a given atomic number $Z$. The maximum of the distribution is found around Mo $(Z=42)$. Right: Plot of mass separation from the relationship between mass $M$ versus mass-over-charge $(M / q)$. The diagonal bands of loci correspond to a given charge state $q$.

The magnetic rigidity $B \rho$ and the angle at the target position are obtained event-by-event from the reconstruction of the trajectories [11, 12]. Together with the measured velocity and the total energy of the fragments, this allows determining both the mass-over-charge $(M / q)$ and the mass $M$ independently. Mass separation is obtained by plotting $M$ versus $M / q$, as seen in Fig. 1. To uniquely select a specific isotope, the atomic number $Z$ and the mass $M$ are selected by placing two-dimensional gates in the $\Delta E-E$ and $M-M / q$ plots, respectively, and by summing events for the various charge states $q$. As an example, the mass distribution for the chain of $\mathrm{Y}$ isotopes is shown in Fig. 2.

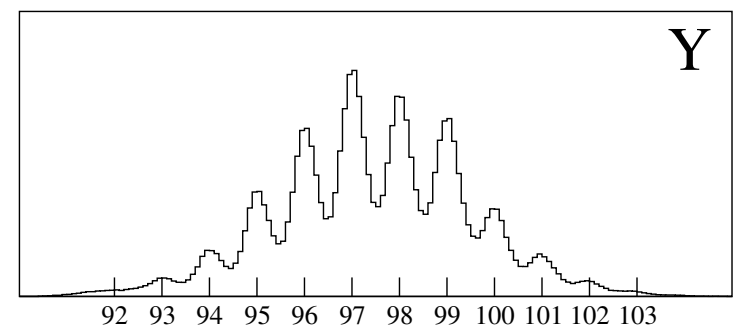

Fig. 2. Mass distribution for the chain of $\mathrm{Y}$ isotopes, summed over all charge states.

The EXOGAM array of segmented Ge clover detectors was used to measure $\gamma$ rays close to the target position. Out of the 11 clover detectors, three were placed at an angle of $135^{\circ}$ with respect to the spectrometer axis, where 
they were sensitive to Doppler shifts, while the remaining detectors were placed at $90^{\circ}$ angles. The Recoil Distance Doppler Shift (RDDS) method [13] was used to extract the lifetimes. The fission fragments exiting from the target were decelerated after a variable distance in a $4.9 \mathrm{mg} / \mathrm{cm}^{2}$ thick $\mathrm{Mg}$ degrader foil. Typical velocities before and after the degrader are $0.12 c$ and $0.10 c$, where $c$ is the speed of light. Those $\gamma$ rays that were emitted from the fission fragment before reaching the degrader foil have a larger Doppler shift compared to those emitted after the degrader, where the velocity is lower. The velocity vector measured in VAMOS after the degrader was used to Doppler correct the energy of the $\gamma$ rays, so the $\gamma$ rays emitted after the degrader appear at the correct transition energy, whereas those emitted before the degrader are shifted to lower energies when observed under backward angles. The lifetime of a state can be extracted from the intensity variation of the two components as a function of distance. Data were taken for seven target-degrader distances ranging from 25 to $1580 \mu \mathrm{m}$, with an average measuring time of approximately 24 hours per distance. During the measurements, the distance was kept constant by a feed-back mechanism based on measuring the capacitance between the two foils. In the RDDS analysis of $\gamma$-ray singles spectra, the feeding time for the state of interest has to be taken into account. The lifetime of a state $i$ that decays with a branching ratio $b_{i j}$ to a state $j$ and is fed with the relative intensity $\alpha_{h i}$ from a state $h$ is found to be as [13]

$$
\tau_{i}(x)=\left(v \frac{d Q_{i j}(x)}{d x}\right)^{-1}\left(Q_{i j}(x)-b_{i j} \sum_{h} \alpha_{h i} Q_{h i}(x)\right),
$$

where $Q_{i j}$ is the relative intensity of the component of the decay transition emitted after the degrader with respect to the total intensity of the transition at the distance $x, Q_{h i}$ the corresponding quantity for the transitions from all feeding states $h$. The denominator contains the velocity $v$ of the fission fragments between the target and the degrader and the derivative of the decay curve $Q_{i j}$ with respect to the distance, which is found by fitting piecewise differentiable polynomials to the data. The velocity, $v$, is determined from the measured velocity after the degrader in VAMOS by considering the energy loss in the degrader foil and by evaluating the energy shift between the two components. To reduce the amount of scattered $\mathrm{Mg}$ ions reaching the focal plane of VAMOS, an additional thin $100 \mu \mathrm{g} / \mathrm{cm}^{2} \mathrm{Au}$ foil was placed at the entrance of the spectrometer, which shifted the charge-state distribution and resulted in a better separation in magnetic rigidity $B \rho$ between the $\mathrm{Mg}$ ions and the fission fragments. 


\section{Lifetime analysis for yttrium isotopes}

The data obtained during the experiment allowed analyzing lifetimes of excited states in a wide range of fission fragments. For even-even isotopes, it was possible to measure previously unknown lifetimes, in particular, for $4^{+}$ and $6^{+}$states in the ground-state bands for several neutron-rich $\mathrm{Sr}, \mathrm{Zr}, \mathrm{Mo}$, $\mathrm{Ru}$, and $\mathrm{Pd}$ isotopes [14]. Here, we will concentrate on the example of ${ }^{99} \mathrm{Y}$, for which the low-lying structure is dominated by a strongly-coupled band of the odd proton in the $[422] 5 / 2^{+}$Nilsson orbital coupled to the strongly deformed ${ }^{98} \mathrm{Sr}$ core [15]. The total $\gamma$ spectrum and partial level scheme for the lowest states in the ground-state band for ${ }^{99} \mathrm{Y}$ is presented in Fig. 3.

\section{${ }^{99} \mathrm{Y}$ total $\gamma$-spectrum}

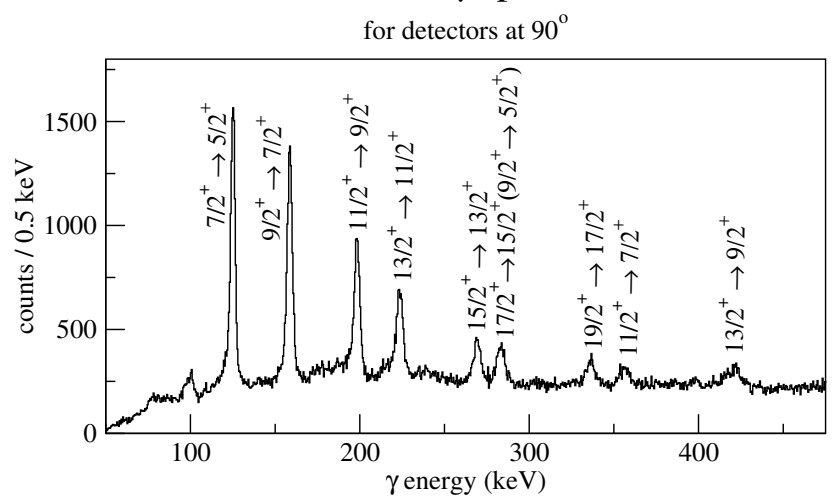

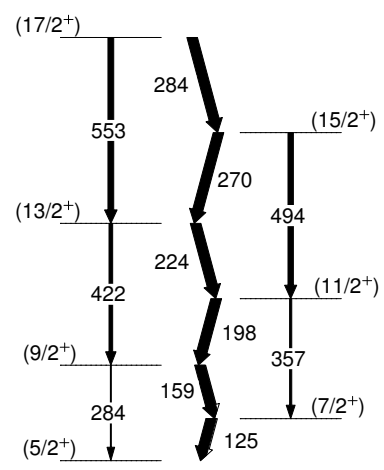

Fig. 3. Left: Plot of the total $\gamma$-ray spectrum for ${ }^{99} \mathrm{Y}$. The spectrum shows the total amount of data, summed over all seven distances, and taken with the Ge detectors placed at $90^{\circ}$, which causes the peaks to be Doppler broadened instead of showing two components with different Doppler shifts. Right: The figure shows the $5 / 2^{+}$ [422] ground state band of ${ }^{99} \mathrm{Y}$.

The strongest peaks in the spectrum correspond to the $\Delta I=1$ transitions, which, in general, have a mixed M1/E2 multipolarity. Weaker $\Delta I=2$ E2 transitions are also visible in the spectrum. The $\gamma$-ray spectra for $\Delta I=1$ transitions from the $9 / 2^{+}, 11 / 2^{+}, 13 / 2^{+}$, and $15 / 2^{+}$states in ${ }^{99} \mathrm{Y}$ for the seven different distances observed at backward angles are shown in Fig. 4. The intensity shift as a function of distance is clearly seen, and lifetimes can be extracted from the analysis of the decay curves using the expression in Eq. (1). 

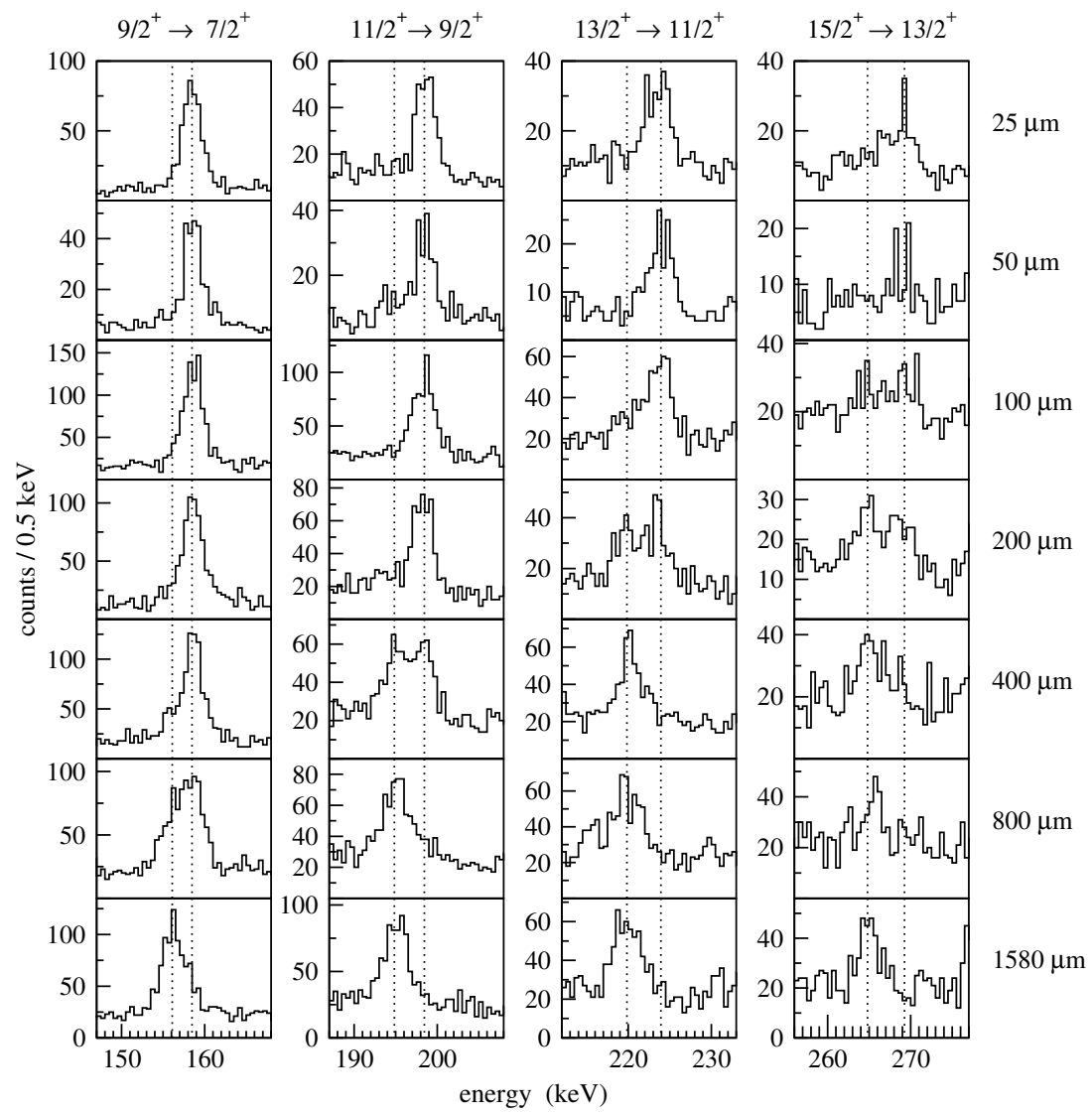

Fig. 4. This figure shows the strongest $\gamma$ transitions in ${ }^{99} \mathrm{Y}$ for the different targetdegrader distances. The dashed lines indicate the energies of the two components.

\section{Outlook and conclusions}

The data for several neutron-rich odd-even isotopes are still under analysis. Preliminary results for ${ }^{99} \mathrm{Y}$ indicate that the lifetimes of the states in the $[422] 5 / 2^{+}$ground-state band are in good agreement with expectations from the particle-rotor model. The branching ratios for the $\Delta I=1$ and $\Delta I=2$ transitions yield the relative $B(\mathrm{M} 1) / B(\mathrm{E} 2)$ strength. The branching ratio together with the lifetime of a given state yield the absolute $B(\mathrm{M} 1)$ and $B$ (E2) strengths, which can be compared to theoretical calculations. In the particle-rotor model, the $B(\mathrm{M} 1)$ and $B(\mathrm{E} 2)$ values are proportional to the $g$-factors $\left(g_{K}-g_{R}\right)^{2}$ and to the intrinsic quadrupole moment $Q_{0}$, respectively. The preliminary results for ${ }^{99} \mathrm{Y}$ indicate that both the quadrupole moments and the $g$-factors are constant within the ground-state band, which 
suggests that the particle-rotor model is an appropriate description. Furthermore, the values are consistent with the measurement of the magnetic moment $\mu$ and the spectroscopic quadrupole moment $Q_{\mathrm{s}}$ for the $5 / 2^{+}$ground state from an experiment using laser spectroscopy [16]. The results support the assigned proton configuration for the ground state and provide a measurement of the deformation of the ${ }^{98} \mathrm{Sr}$ core. The lifetime measurement for ${ }^{99} \mathrm{Y}$ is, therefore, complementary to a recent Coulomb excitation experiment which investigated the shape and shape coexistence of the low-lying states in ${ }^{98} \mathrm{Sr}$ using a radioactive beam from the ISOLDE facility at CERN [5].

The recoil-distance Doppler shift technique was successfully applied for the first time in combination with fusion-fission reactions in inverse kinematics. A large number of previously unknown lifetimes can be determined under identical experimental conditions from the same experiment. The method is particularly powerful as it allows event-by-event identification of the fission fragments. The data is still under analysis, in particular, for the more complex odd-mass isotopes of $\mathrm{Y}, \mathrm{Nb}$, Tc, and $\mathrm{Rh}$, and more specific results will be published in forthcoming publications. Preliminary results for the odd-mass $\mathrm{Y}$ isotopes with $N>60$ suggest that the ground-state bands can be well-described with the particle-rotor model with a constant quadrupole moment.

\section{REFERENCES}

[1] I. Agneli, K. Marinova, At. Data Nucl. Data Tables 99, 69 (2013).

[2] S. Naimi, Phys. Rev. Lett. 105, 032502 (2002).

[3] T. Kibedi, R.H. Spear, At. Data Nucl. Data Tables 89, 77 (2005).

[4] J.L. Wood, Nucl. Phys. A 651, 323 (1999).

[5] E. Clément et al., EPJ Web Conf. 66, 02021 (2014); Phys. Rev. Lett. 116, 022701 (2016).

[6] C. Sotty et al., Phys. Rev. Lett. 115, 172501 (2015).

[7] H. Mach et al., Phys. Rev. C 41, 1141 (1990).

[8] J.H. Hamilton et al., Prog. Part. Nucl. Phys. 35, 635 (1995).

[9] A.G. Smith et al., Phys. Rev. C 86, 014321 (2012).

[10] Y.X. Luo et al., J. Phys. G: Nucl. Part. Phys. 31, 303 (2005).

[11] S. Pullanhiotan et al., Nucl. Instrum. Methods A 593, 343 (2008).

[12] S. Pullanhiotan et al., Nucl. Instrum. Methods B 266, 4148 (2008).

[13] A. Dewald et al., Z. Phys. A 334, 163 (1989).

[14] L. Grente et al., Ph.D. Thesis, University Paris Sud, 2014 to be published.

[15] R.A. Meyer et al., Nucl. Phys. A 439, 510 (1985).

[16] B. Cheal et al., Phys. Lett. B 645, 133 (2007). 